\title{
Self-diagnostic approach for cell counting biosensor
}

\author{
Qais Al-Gayem ${ }^{1}$, Hussain F. Jaafar ${ }^{2}$, Saad S. Hreshee ${ }^{3}$ \\ ${ }^{1,3}$ Department of Electrical Engineering, University of Babylon, Iraq \\ ${ }^{2}$ Department of Biomedical Engineering, University of Babylon, Iraq
}

\begin{tabular}{|c|c|}
\hline Article Info & ABSTRACT \\
\hline Article history: & In this research, a test monitoring strategy for an array of biosensors is \\
\hline Received Oct 12, 2020 & proposed. The principle idea of this diagnostic technique is to measure and \\
\hline Revised Mar 29, 2021 & online health monitoring technique at the system level. The work includes \\
\hline Accepted Apr 27, 2021 & $\begin{array}{l}\text { implementation of the diagnostic system, system architecture for analogue } \\
\text { part, and SNR analysis. The technique has been applied on a cell coulter }\end{array}$ \\
\hline Keywords: & $\begin{array}{l}\text { counting biochip where the design and fabrication of this sensing chip with } \\
\text { electrodes make the coulter counter be an effective mean to count and }\end{array}$ \\
\hline $\begin{array}{l}\text { Biochips } \\
\text { Biofluidic } \\
\text { MEMS }\end{array}$ & $\begin{array}{l}\text { analyses the cells in a blood sample. The experimental results show that the } \\
\text { indication factor of the sensing electrodes has increased from } 1 \text { to } 1.8 \\
\text { gradually depending on the fault level. }\end{array}$ \\
\hline
\end{tabular}

Self-test

Sensors

This is an open access article under the CC BY-SA license.

Corresponding Author:

Qais Al-Gayem

Department of Electrical Engineering

University of Babylon

Babil, Iraq

Email: eng.qais.karem@uobabylon.edu.iq

\section{INTRODUCTION}

Micro electro mechanical systems (MEMS) have developed and expanded enough to meet the complex needs of biomedical science. Biochip is one of these microsystems which provide the ability to accurately detect and separate biological particles from blood and other fluids. This is very essential in diagnosis, healthcare, and research. Several micro devices are already developed, like micro-electrodes, micro-needles, and advances biosensors (enzyme field effect transistors) [1]-[11]. In recent years, systems called Lab-On-a-Chip have been developed to be capable of performing a full set of bio-chemical analysis and reactions on a single platform, which includes sample collection and handling, physical and chemical processes, and signal detection [1]. Utilization of nano electro mechanical systems (NEMS) could also result in reduction of samples and reagents, and improvement in analytical performance. This makes the analysis safe, easy to be realized, and accurate. Detection of ammonia could be achieved using a microfluidic device, where a pair of electrodes was used to measure the capacitance of the river water to detect the ammonia [2]. In another work, the design of a remote monitoring device for biomedical field was presented [3]. In this work, the system includes four biomedical sensors and high accuracy results have been obtained. Numerical study and optimization of $\mathrm{KOH}$ wet etching process were proposed to achieve the desired shape and size of MEMS diaphragm [4]. The results of this method offered an effective technique to realize the chosen design. Characterization of the geometrical shape of electrodes for dielectrophoresis was presented where the particles distribution has been evaluated by analyzing the particles behavior in the existence of non-uniform electric field [5]. 
Detection and classification of cells are important aspects in medical research for diagnosis and treatment of diseases at cellular level. Cell counting techniques are widely used in laboratory and pathology. In the literature, few techniques are designed based on cytometry, fluorescence, and coulter counter. Flow cytometry was used to count and investigate microscopic particles, such as cells and chromosomes, and is based on suspend the cells in a stream of fluid and passing them by electronic controlling and detection circuits [6]. It allows simultaneous multiparametric analysis of physical or chemical characteristics of up to thousands of particles per second. A special type of flow cytometry is fluorescence-activated cell sorting (FACS). It offers a technique for arranging a heterogeneous mixture of bio-logical cells into different containers, one cell at a time and based on the fluorescent characteristics of each cell [7]-[8]. The cell suspension is focused in the center of a narrow and fast flowing stream of liquid. The movement is organized so that there is a very large separation between cells and by using a vibrating mechanism, the stream of cells is divided into separated droplets. After that, the flow crosses the fluorescence measuring site where the fluorescent character of the cell of interest is recorded. Another effective cell counting method based on a coulter counter has been used in this work. Wallace H. Coulter developed a technique for counting and sizing particles using impedance measurements. It was mainly developed to count blood cells by measuring the changes in electrical conductance as cells suspended in a conductive fluid passed through a small hole [9]. Cells, being non-conducting particles, could alter the effective cross-section of the conductive micro channel. If these particles are non-conducting, the electrical resistance across the channel increases, causing the electric current passing across the channel to slightly decrease. By monitoring such pulses in electric current, the number of particles for a given volume of fluid can be counted. When a particle passes, there is a change in the electrical resistance of the fluid between the electrodes and that causes a pulse. Assuming the particles have infinite resistance, the change in the electrical cross section depends on the cross section of the particle. The change in the electrical resistance can be determined by following the method, presented in [10]-[11].

Due to growing interest in this type of biochips, it becomes essential to increase the reliability of these microsystems and avoid the false readings. This can be achieved by developing self-test methodologies that can be employed in the system design to make the output reading more reliable. Different techniques have been proposed and developed and here is a review about this topic. Embedded test has become the important part of the design flow of Bio-MEMS. In this technique, dependability has been adopted in the microsystem and its medical applications where the sources of the malfunction are still unknown while the whole interconnect subsystem of the biochips has been tested and different procedures for characterising the mechanical and fluidic machinery have been proposed [12]-[13]. The potential of using oscillation based test (OBT) has been investigated, where this technique was utilized to generate indication about the status of the electrodes in multi electrode array (MEA) [14]-[16]. This technique produces an oscillation frequency that is dependent on the fault level on the surface of the sensing electrode. This fault level affects the capacitance of the electrical double layer that is generated on interface layer between the testing liquid and the top sensing layer. A built-in-self-test (BIST) architecture for micro electrode dot array (MEDA) which is an example of digital biochips has been proposed in a research where defects in the MEDA can be detected and the faulty microcells can be easily identified [17]. Another approach which includes the test of the microfluidic parameters and the CMOS circuitry, where a pass/fail indicator, was generated to ensure ideal functional operation of the different chip units [18]. A work which is related to digital biochips has also been presented, where techniques for test planning, fault classification, and test optimization were proposed [19]. The main and soft fault have been identified and detected by tracking the motion of droplets using an RC oscillator [20]-[21]. The open structure of the digital array can be easily reconfigured to avoid the defected cells and run a biochemical sensing operation and fault recognition scheme simultaneously. In another work, a BIST for the membrane of piezoresistive MEMS sensor was presented [22]. The importance of the proposed method is in analyzing the output response of the sensor after applying an on chip signal on it (as a test stimulus). The simulation results proved that this technique is very effective and can be used as alternative for the external circuits to reduce the manufacturing cost. In addition, logic BIST with variable test clock generation was used to observe the aging phenomenon on a chip under accelerated normal life test [23]. Digital temperature and voltage sensors were used and the experimental results showed a significant increase in the circuit delay. Moreover, the failure modes of capacitive MEMS microphone were detected by using a technique which is based on acoustic measurements for the pull-in and snapback voltage ranges [24]. The results showed a significant rate in detecting membrane capture events for this type of device.

The work presented in this research is motivated from the ability to produce accurate measurements when using very small sample at very low cost and is focusing on biochip electrodes that are used to count the cells in a liquid sample. These electrodes can be easily degraded due to many reasons, such as losing the surface material (sensing layer) during the normal operation and the chemical reactions or settling some biomaterials on this surface. The failure modes, related to these reasons of degradation are: a considerable increase in the electrode impedance, a fluctuation in the interface liquid/solid capacitance, and a reduction in 
the SNR of the extracted signal. The theory of the electrode/electrolyte interface and the electrical properties of this interface are crucial parameters within the design of the test technique and the background is available in [25]-[28]. As a consequence, all the failure mechanisms will directly affect the electrode conductance and accordingly the sensing of the biochip. Therefore to reduce the need for external circuits and then the manufacturing cost and increase the reliability of this biochip, it is essential to have an embedded self-check for the whole electrode array. This is achieved by the proposed technique where the impedance of the electrodes are measured and compared with reference impedance to locate the partially and fully degraded electrodes.

\section{RESEARCH METHOD}

In this section, the test methodology for the whole systems is proposed.

\subsection{System architecture}

As shown in Figure 1, the proposed system consists of a control and processing unit, a switching matrix, and a testing circuit. The testing circuit is made up by a half bridge circuit, namely two identical resistors and an $\mathrm{AC}$ signal $(1.5 \mathrm{mV})$ at a frequency of $1 \mathrm{kHz}$. The bridge circuit is used to measure the impedance change of the electrodes. The switching matrix contains an analogue switch array and a bidirectional analogue multiplexer array. The former is used to control the connection/isolation between the electrodes and user interface, while the later is to switch the electrodes from different positions.

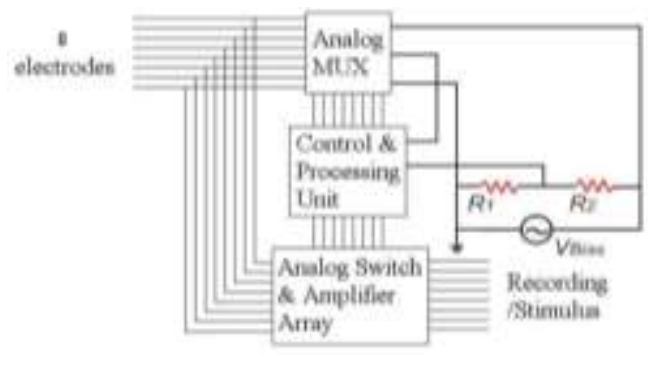

Figure 1. System architecture

The control and processing unit is responsible for synchronization of the whole system and calculation of the relative impedance change. As VBias is actually an AC signal, information about impedance change has to be extracted from AC signals. Hence as shown in Figure 2, a method similar to the amplitude shift keying (ASK) decoding mechanism is adopted.

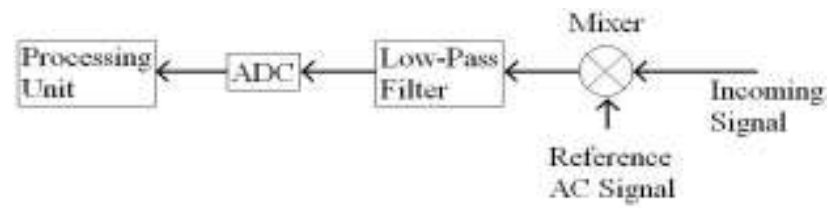

Figure 2. Block diagram of a pre-processing unit

The mixer will produce a DC signal and the second harmonic of the original signal, as expressed in (1):

$$
a \cos (\omega t) \cos (\omega t)=a \frac{1+\cos (2 \omega t)}{2}
$$

Hence the impedance change information (as represented by (1)) will be carried by the DC voltage level. A low-pass filter can be used to isolate the DC signal and the second harmonic signal, and finally an analogue to digital converter is used to digitalize the impedance change information. The system provides online monitoring of the degradation states of electrodes, and is triggered by a clock signal which has low duty cycle and frequency. When the clock signal is logic high, the system is performing impedance tests. The 
analogue multiplexers are on and analogue switches are turned off. Testing data will be delivered to the processing unit and diagnosis will be performed. During the second half while the clock signal is logically low, the chip is in use. The analogue switches will be turned on and the multiplexers are off. If an electrode is detected to be degraded in the previous half, the corresponding switch will be off so that the wrong experimental data will not be delivered to the user.

The system, introduced in this section, will turn off the corresponding switch if an electrode is detected to be degraded, which means fewer electrodes are in use. An advanced system is able to compensate for the degradation, hence the number of electrodes, in use, will maintain unchanged even the electrodes are degraded. The used amplifier is a voltage controlled amplifier whose gain and offset are changeable. The previous works have showed that the degradation can be modelled by the resistance change which directly affects the magnitude and offset of the electric signals. Hence, by adjusting the gains and offsets of the amplifier, compensation for the damage of electrodes can be achieved.

\subsection{Switch and amplifier design}

It can be noted in Figure 1 that the analogue part is separated from the digital part, while the analogue part is in direct contact with the signals. Hence one of the crucial questions is how to achieve analogue switch and amplifier with good quality. Low noise, low cross-talking, high sensitivity, and high resolution are required in this design (As the bi-directional analogue multiplexer adopts a similar working mechanism as the analogue switch, the design of this component can be seen equivalent to that of the switch).

The design is demonstrated by using Cadence, and a single NMOS transistor is used as the switch. Some applications of biochips have indicated that the electrical signals coming from the cultured cells or tissues can be very small to be few micro volts [6]-[8]. Hence in order to achieve good sensitivity and resolution, the switch should be able to handle a signal at nano-volt level. Thus the input signal, namely the signal from electrode, is assumed to have a magnitude of $5 \mathrm{nV}$. Two different technology files (C35B4C3 and SMIC018) are used and the results will be presented respectively. The AMS of 0.35 micron fabrication process, modeled by technology file C35B4C3, was proposed by Austria Micro System Corporation, with minimum channel length of 0.35 micron. Figure 3 shows the performance of the switch. The channel length and width of the NMOS transistor are $350 \mathrm{~nm}$ and $3 \mu \mathrm{m}$ respectively.

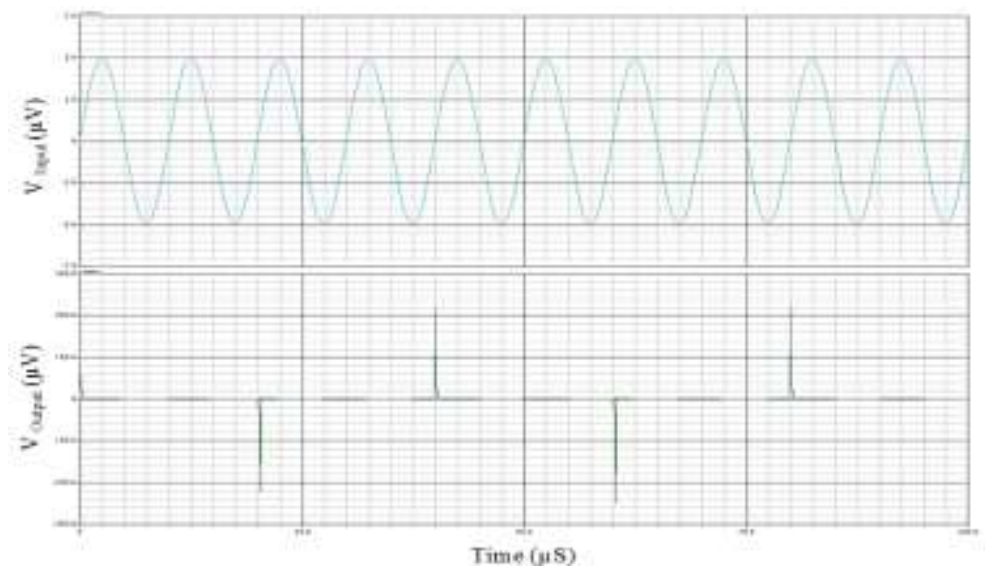

Figure 3. Analogue switch performance (input and output)

The following points can be noted from the Figure 3: In the above simulation, the gate of the transistor is connected to a clock signal. Hence whenever the clock signal transits from high to low or from low to high, an unwanted transient response takes place which can be as large as few $\mu \mathrm{V}$. The magnitude of this transient response depends on the fall/rise time of the clock signal. Figure 4 shows the responses with 5 different fall/rise time values. The transient response becomes smaller as the fall/rise time increases. In this bio-fluidic diagnostic system, since the switches are not designed to switch-on and switch-off frequently, the large transient response is acceptable. Good isolation is achieved when the transistor is off. When the transistor is on, the "on" resistance of the transistor and the load form a voltage divider, and the signal is degraded. Figure 4 depicts the signal degradation with respect to frequency for different channel widths. The load resistance is set to be $10 \mathrm{~K} \Omega$ (usually the input resistance of the amplifier is larger than this value). A channel width of $20 \mu \mathrm{m}$ gives a signal degradation of $0.072 \mathrm{~dB}$. 


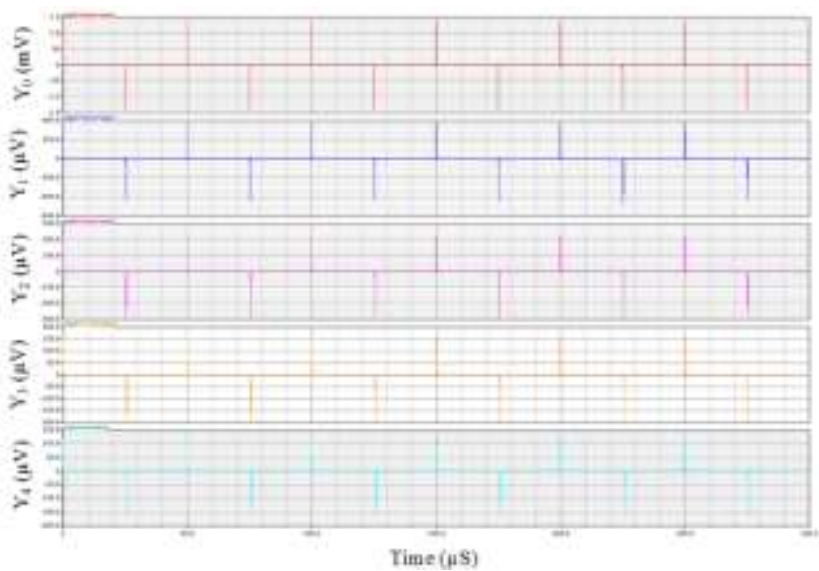

Figure 4. Transient responses with respect to five different rise/fall times

Another fabrication process, modeled by technology file "SMIC018", is tried. This fabrication process is proposed by Semiconductor Manufacturing International Corporation, with minimum channel length of $180 \mathrm{~nm}$. Figure 5 shows the performance of the corresponding switch.

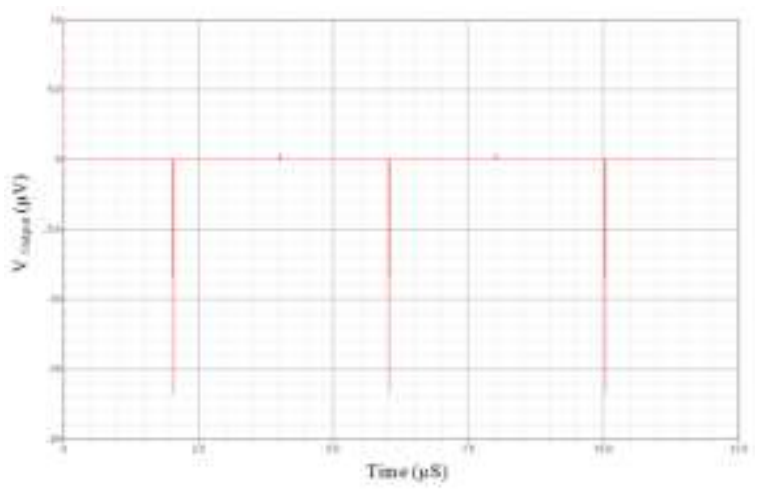

(a)

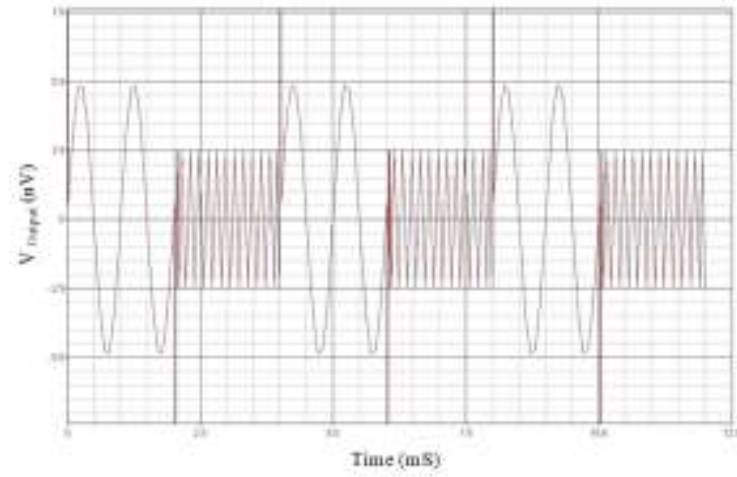

(b)

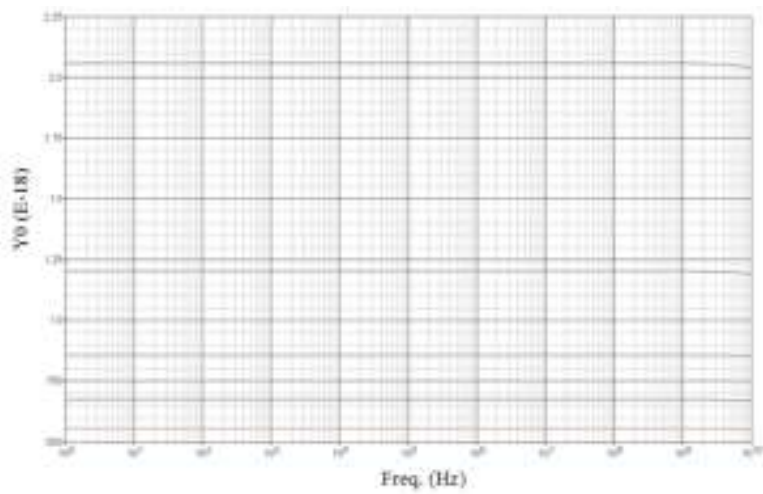

(c)

Figure 5. Analogue switch performance: (a) output signal, (b) a closer look at the output signal, and (c) noise analysis

In this transistor, noise is still not a major problem. As shown in Figure 5(c), the power spectral density is even smaller than that implemented by the fabrication process of AMS of 0.35 micron. However, when the transistor is off, as presented in Figure 5(b), the static cross-talking can be very large. The 
magnitude is in the same order with the incoming signals. Thus this fabrication process cannot achieve the necessary requirements. The amplifier design is implemented by using commercially available operational amplifier ICs. As the DC biasing and the incoming signals are isolated by capacitors in a BJT or FET amplifier, DC signals or low frequency signals cannot be amplified linearly. Hence op-amp is the only choice to implement the amplifier. Figure 6 shows the amplifier design.

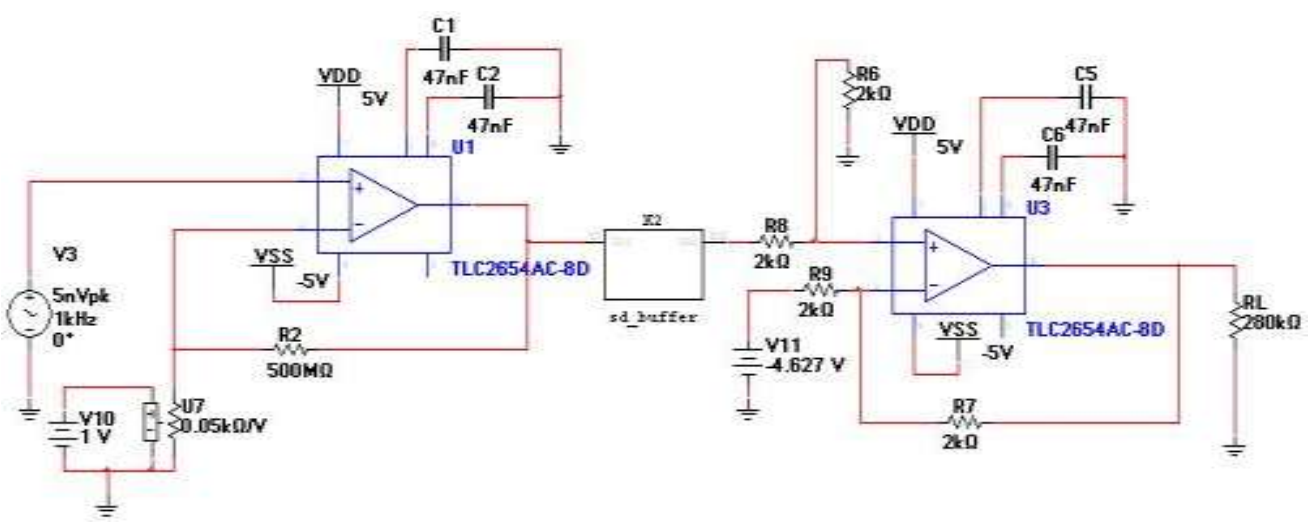

Figure 6. The amplifier configuration

The amplifier consists of three parts: a non-inverting amplifier, a differential amplifier, and a buffer in between. The non-inverting amplifier is used to amplify the signal, while the differential amplifier is responsible for eliminating the offset introduced by the op-amps. It should be noted that the gain of this amplifier depends on the ratio of resistors R2 and U7, and the offset level depends on the voltage source V11. Hence the compensation for electrode degradation can be implemented by adjusting the values of resistor U7 and voltage source V11.

To summary this section, analogue switch and amplifier designs are presented. AMS of 0.35 micron fabrication scheme is a potential candidate to achieve the required transistors with acceptable signal degradation at switch on state and good isolation at switch off state. It also achieves good signal-to-noise ratio. Amplifier can be implemented by commercially available ICs, which implies to the feasibility of integrating the analogue part of the diagnostic system.

\section{APPLICATION OF THE DIAGNOSTIC APPROACH ON THE CELL COUNTING BIOSENSOR}

Mixing, focusing, and measurement are the main three parts of the biosensor, and the fluid passes sequentially through them. Two fluids are made to pass through square wave shaped channel to ensure chaotic mixing. This occurs due to turbulence and maintains a proper amount of osmolality. The uniformity in the impedance gives accurate results during measuring the changes at the measuring section. For accurate cell count, it is necessary for the cells to pass one at a time through the measuring electrodes. For this reason the fluid passes through a narrow focusing region. This region is connected to two electrodes, and these two electrodes create a negative field around the focusing region to get a phenomenon called dielectrophoretic (DEP) focusing. The DEP force, acting on the particles, makes the cells enter the narrow width channel one by one without clogging. After passing the focusing channel, a straight streamlined flow of single cells is produced. These cells travel one by one to the series of measuring electrodes. These electrodes produce an electric field in the direction perpendicular to the plane of the channel. As the cells pass through the electrodes, the impedance change generates a pulse. This pulse has the same voltage for the same type of cells. The output of each electrode is connected to an oscilloscope and PC using LabView software to obtain a real time graph. The graph shows various peaks for a particular type of cell. The data is captured using data acquisition card and stored. By calculating the number of peaks, the number of particular type of cells could then be obtained.

The sensor chip was designed using AutoCAD and then transferred to actual platform using x-ray exposure and development. The chip comprises of a lid layer and base layer. The lid layer is a 2mm PMMA electrode array and the base layer is SU-8 coating patterned and cured to form fluid channels. These layers 
are drilled with holes for fluid and electrode access before being thermally bonded together to form the complete laminate.

The fabrication process starts with fabrication of electrodes where film based conductive carrier is coated with $6 \mu \mathrm{m}$ SU-8 and photo patterned with emulsion on an acetate photo tool. A 2 mm PMMA panel is plasma treated and coated with a $10 \mu \mathrm{m}$ layer of transfer adhesive and electrode array is cold laminated to the surface of this panel. The transfer adhesive is then activated by exposing to UV through the PMMA and is then thermally cured. The base electrode layer is plasma treated and coated with $25 \mu \mathrm{m}$ of SU-8 photoresist using emulsion on acetated photo tooling. Fluid and electrode access holes are drilled in registration with electrode and fluidic features. The lid and base layers are assembled in bonding jigs and exposed to temperature and load to achieved bonded laminate. Finally, bonded laminate has fixed holes drilled and is diced up into individual components. Figure 7 shows the final biosensor.

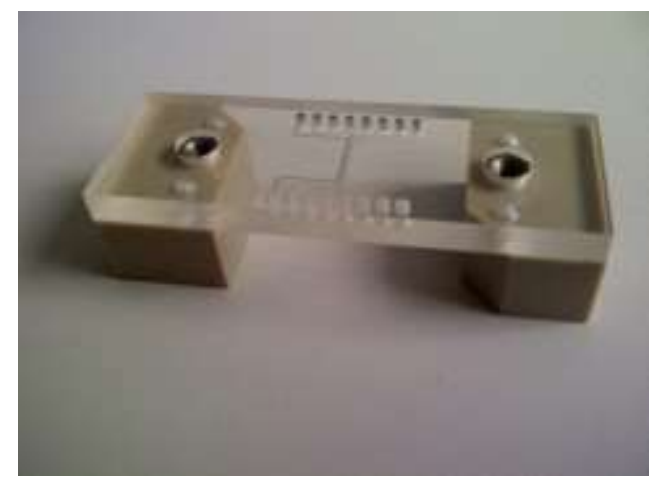

Figure 7. The final fabricated biosensor placed on platform

\section{TEST RESULTS}

The inlets and outlets are connected to the chip via small diameter pipes with connectors at the end of each of them. The fluid is pumped in the inlets by pumps which have injectors on them. Pump speeds can be easily controlled and is maintained quite low, and is $1.5 \mu \mathrm{l} / \mathrm{s}$. The slow speed ensures accurate mixing and laminar flow. Another pipe is connected to the outlet to take out the fluid after the measuring process. An electrical circuit is required to measure and interpret the change in the impedance of the fluid. This circuit takes the output from the electrodes and amplifies it via differential amplifier. Figure 8 shows the experimental setup.

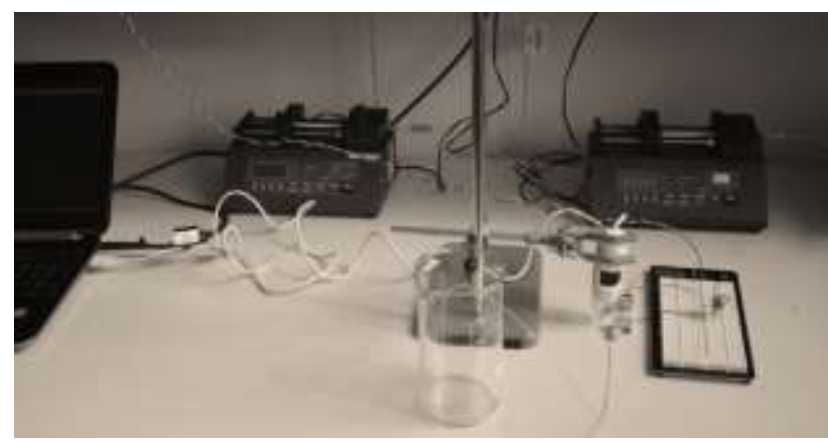

Figure. 8 The experimental setup

After the setup of the hardware components, the fluidic chip has to be tested. The flow of particles and the impedance changes in form of voltage peaks were tested in the focusing and measuring regions. The DEP electrodes are used to make up the focusing region, so that the cells are focused into single line and to the center of the channel. An AC electric field is applied across the electrodes, since application of DC field will cause the charge to keep on accumulating and lead to damage of the channels. Micro-beads of 
approximately $14 \mu \mathrm{m}$ are injected into the channels. The size of the micro-beads is so chosen because it is equivalent to the size of WBCs, which are the biggest cells in the blood. A sinusoidal wave of $6 \mathrm{~V}$ peak amplitude and $12 \mathrm{MHz}$ frequency is applied to the focusing electrodes. The micro-beads were aligned on one line and travelled down the channel one by one.

The output from the electrode was taken into a measuring circuit which was connected to the oscilloscope. The measuring circuit converts the impedance changes into electrical changes which is passed through a filter and then amplified by the instrumentation amplifier. The inlets were injected with microbeads of different specific sizes as $5 \mu \mathrm{m}, 10 \mu \mathrm{m}$, and $15 \mu \mathrm{m}$, and these sizes are selected because they are equivalent to platelets RBCs and WBCs. Different micro-beads provided different amplitudes which were visible on the oscilloscope. In Figure 9, the peaks of $0.5 \mathrm{~V}$ are corresponding to $5 \mu \mathrm{m}$ sized beads, and similarly the peaks of $1.4 \mathrm{~V}$ and $3.2 \mathrm{~V}$ are corresponding to $10 \mu \mathrm{m}$ and $15 \mu \mathrm{m}$ sized beads respectively. To evaluate the test methodology, different levels of degradation have been emulated at the sensor electrodes (by applying a stress signal for different periods) and the test scheme was applied where the impedance is measured with respect to the reference impedance.

The reference impedance has been defined as the average value of the impedance readings for the whole electrode array before running any test on the biochip. To study and consider the effect of degradation on the electrode impedance, the biochip has been used for eight normal operation cycles and the impedance of each electrode was measured after each cycle. The electrodes in the biochip can be easily degraded due to many reasons during the normal operation, such as losing the surface material (sensing layer) due to chemical reactions or settling some bio-materials on this surface. This will directly increase the measured impedance. Table 1 shows the extracted data after each cycle where a considerable increase in the electrode impedance (Electrode No. 1, 4, and 5) can be seen. To estimate the level of failure, the measured impedance data for all cycles were divided by the reference impedance and an indication has been generated about the states of each microelectrode in the biochip. Figure 10 shows the increase in the impedance for fault-free and faulty electrodes. The readout of the test scheme is presented in Figure 11 where it can be noted that the faulty electrode can be easily located and the level of fault can also be calculated by dividing the measured impedance on the reference impedance (indication factor). This indication factor is ranged between 1.1 for the partially degraded electrode and around 1.8 for the fully degraded electrode.

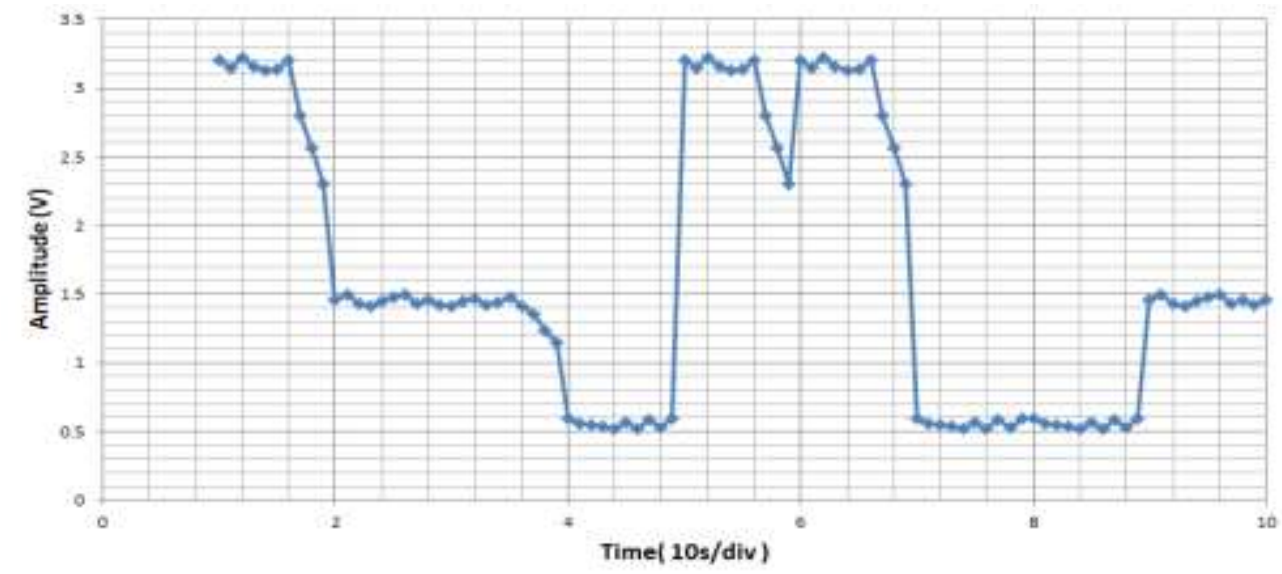

Figure 9. The biosensor recorded data

Table 1. The impedance measurements

\begin{tabular}{|c|c|c|c|c|c|c|c|c|}
\hline $\begin{array}{c}\text { Electrode } \\
\text { No. }\end{array}$ & $\begin{array}{c}\text { Impedance } \\
(\mathrm{K} \Omega) \\
\text { First cycle }\end{array}$ & $\begin{array}{c}\text { Impedance } \\
(\mathrm{K} \Omega) \\
\text { Second cycle }\end{array}$ & $\begin{array}{l}\text { Impedance } \\
(\mathrm{K} \Omega) \\
\text { Third cycle }\end{array}$ & $\begin{array}{l}\text { Impedance } \\
(\mathrm{K} \Omega) \\
\text { Forth cycle }\end{array}$ & $\begin{array}{c}\text { Impedance } \\
(\mathrm{K} \Omega) \\
\text { Fifth cycle }\end{array}$ & $\begin{array}{c}\text { Impedance } \\
(\mathrm{K} \Omega) \\
\text { Sixth cycle }\end{array}$ & $\begin{array}{c}\text { Impedance } \\
(\mathrm{K} \Omega) \\
\text { Seventh cycle }\end{array}$ & $\begin{array}{c}\text { Impedance } \\
(\mathrm{K} \Omega) \\
\text { Last cycle }\end{array}$ \\
\hline 1 & 52 & 56 & 63 & 71 & 75 & 81 & 84 & 88 \\
\hline 2 & 50 & 52 & 52 & 54 & 55 & 55 & 57 & 58 \\
\hline 3 & 52 & 55 & 53 & 57 & 57 & 57 & 58 & 60 \\
\hline 4 & 49 & 53 & 61 & 70 & 73 & 77 & 84 & 85 \\
\hline 5 & 51 & 56 & 63 & 69 & 70 & 71 & 73 & 73 \\
\hline 6 & 51 & 55 & 54 & 57 & 56 & 56 & 59 & 59 \\
\hline 7 & 50 & 51 & 51 & 54 & 55 & 56 & 57 & 57 \\
\hline 8 & 51 & 52 & 52 & 53 & 55 & 56 & 59 & 61 \\
\hline
\end{tabular}




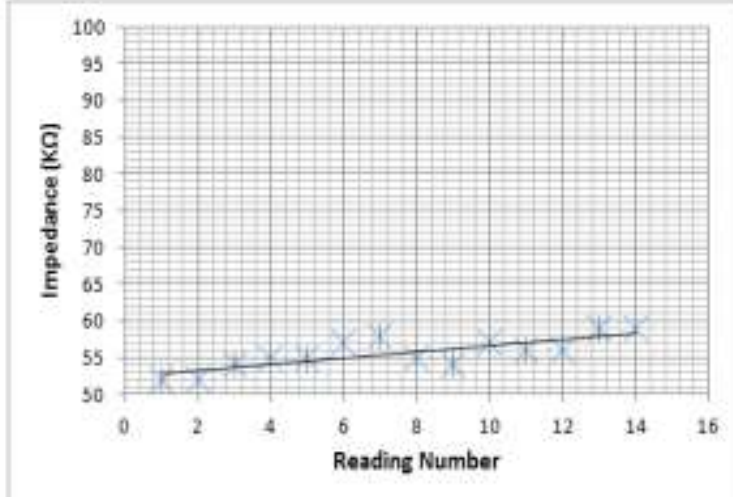

(a)

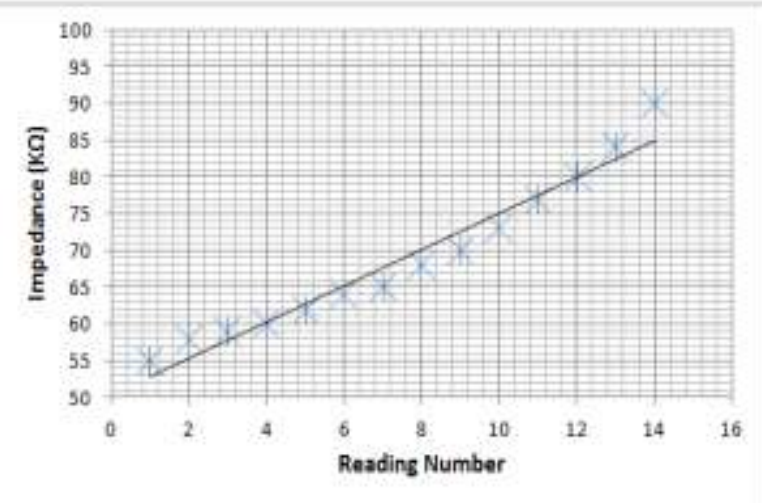

(b)

Figure 10. The impedance measurements for (a) fault-free electrode, (b) faulty electrode

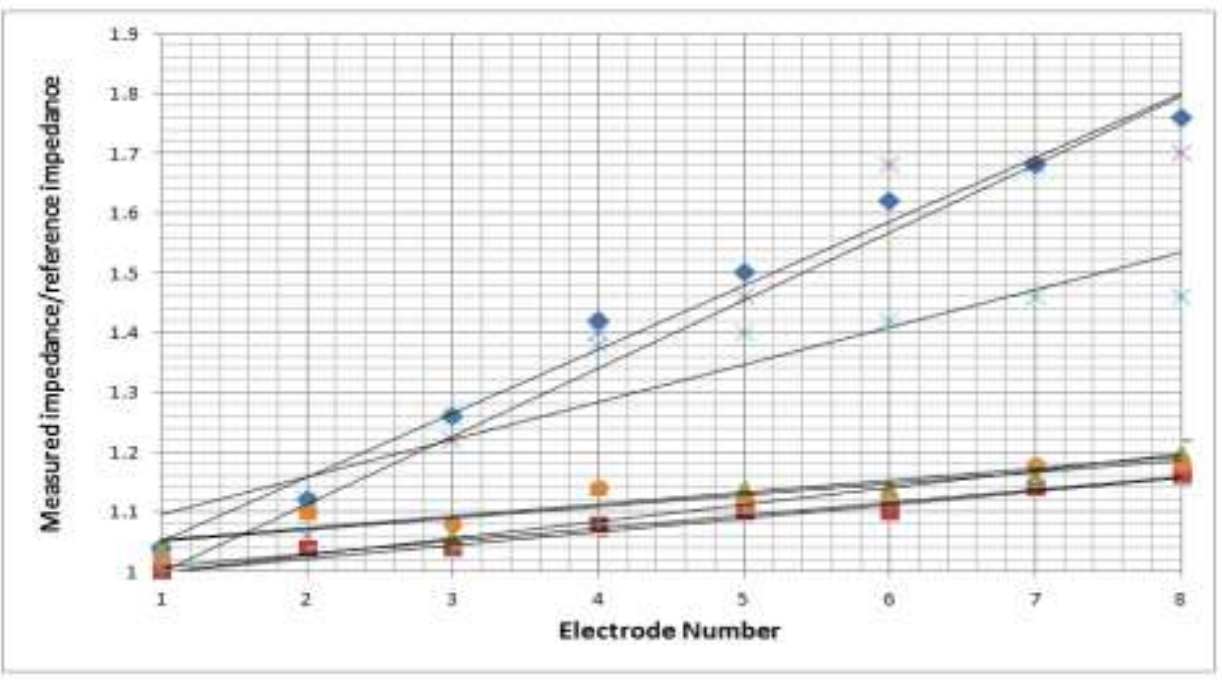

Figure 11. The readout (indication factor) of the test scheme

\section{CONCLUSION}

Robust microfluidic operations and high confidence readings are main challenges that are facing developing the portable micro-biochemical sensing procedures. In this work, a test monitoring methodology for a cell counting sensor is presented. The methodology is based on measuring and comparing the electrode impedance of an array of biosensors. The system consists of a control and processing unit, a switching matrix and a testing circuit. The system provides on-line monitoring of the degradation states of whole array, and is triggered by a clock signal which has low duty cycle. When the clock signal is logic high, the system is performing impedance tests, while when it is logic low, the biochip is in use. The work adopted studying the analogue switch and the amplifier design, and the noise analysis of the system. The test scheme was applied on a coulter counter which is used to analyse the cells in a blood sample and promising results have been extracted showing that this methodology can be utilized to increase the reliability in next generation biochips. The future work will be investigating the ability to utilize a compensating technique for the partially degraded electrodes to enhance the overall performance of the system. In addition, the use of this approach as a prognostic technique will be considered to calculate the remaining useful life of the biochip.

\section{ACKNOWLEDGEMENTS}

This work was supported by Ministry of Higher Education and Scientific research in Iraq, and University of Babylon. 


\section{REFERENCES}

[1] N. Piacentini, et al., "MEMS-based Blood Cell Counting System," 15th IEEE International Conference on Electronics, Circuits and Systems, St. Julien's, pp. 198-201, 2008, doi: 10.1109/ICECS.2008.4674825.

[2] M. M. Ramli and M. R. Ahmad, "Capacitance-based Microfluidic Device for Early Ammonia Detection in River Water," Indonesian Journal of Electrical Engineering and Computer Science (IJEECS), vol. 20, no. 2, pp. 761767, Nov 2020, doi: 10.11591/ijeecs.v20.i2.pp761-767.

[3] N. A. A. Rahman and A. B. Jambek, "Biomedical Health Monitoring System Design and Analysis," Indonesian Journal of Electrical Engineering and Computer Science (IJEECS), vol. 13, no. 3, pp. 1056-1064, March 2019, doi: 10.11591/ijeecs.v13.i3.pp1056-1064.

[4] N. Yusof, et al., "Optimization of KOH Etching Process for MEMS Square Diaphragm Using Response Surface Method," Indonesian Journal of Electrical Engineering and Computer Science (IJEECS), vol. 15, no. 1, pp. 113121, Jul 2019, doi: 10.11591/ijeecs.v15.i1.pp113-121.

[5] N. Burham, et al. "Characterizations of Electrode Geometrical Shape for Dielectrophoresis," Indonesian Journal of Electrical Engineering and Computer Science (IJEECS), vol. 15, no. 2, pp. 671-677, Aug 2019, doi: 10.11591/ijeecs.v15.i2.pp671-677.

[6] A. L. Givan, "Flow Cytometry First Principle", Book, 2001.

[7] L. Herzenberg, et al., "Interpreting Flow Cytometry Data: A Guide for the Perplexed," Nat Immunol, vol. 7, pp. 681-685, 2006, doi: 10.1038/ni0706-681.

[8] A. Fu, et al., "A Microfabricated Fluorescence-activated Cell Sorter," Nat Biotechnol, vol. 17, pp. 1109-1111, 1999, doi: 10.1038/15095.

[9] Y. Wu, et al., "MEMS Coulter Counter for Dynamic Impedance Measurement of Cells," SENSORS, 2011 IEEE, Limerick, pp. 794-797, 2011, doi: 10.1109/ICSENS.2011.6127238.

[10] M. Hügle, et al., "A Lab-on-a-Chip for The Extraction of Bacterial Nucleic Acids From Whole Human Blood," 20th International Conference on Solid-State Sensors, Actuators and Microsystems \& Eurosensors, pp. 925-927, 2019, doi: 10.1109/TRANSDUCERS.2019.8808273.

[11] D. Lizanets and R. Walczak, "Algorithms and Approaches for Processing an Output Signal from Lab-on-a-chip Devices in Bioanalysis," XII International Conference on Perspective Technologies and Methods in MEMS Design (MEMSTECH), pp. 6-8, 2016, doi: 10.1109/MEMSTECH.2016.7507507.

[12] H. G. Kerkhoff, "Testing of MEMS-based Microsystems," Proceedings of 10th IEEE European Test Symposium, pp. 223-228, 2005, doi: 10.1109/ETS.2005.40.

[13] B. L. Gray, et al., "Mechanical and Fluidic Characterization of Microfluidic Interconnects for Lab-on-a-chip Applications," Proceedings of 14th IEEE International Mixed-Signal Test Workshop, 2008, doi: 10.1109/IMS3TW.2008.4581624.

[14] Q. Al-Gayem, et al., "Built-in Test Solutions for the Electrode Structures in Bio-fluidic Microsystems," Proceedings of the IEEE European Test Symposium, pp. 73-78, 2009, doi: 10.1109/ETS.2009.24.

[15] Q. Al-Gayem, et al., "An On-line Monitoring Technique for Electrode Degradation in Bio-fluidic Microsystems," IEEE International Test Conference, Austin, TX, 2010, doi.org/10.1109/TEST.2010.5699269.

[16] H. Khan, et al., "A Housekeeping Prognostic Health Management Framework for Microfluidic Systems," IEEE Transactions on Device and Materials Reliability, vol. 17, no. 2, pp. 438-449, 2017, doi: 10.1109/TDMR.2017.2694227.

[17] Z. Li, et al., "Built-in Self-test for Micro-electrode-dot-array Digital Microfluidic Biochips," IEEE International Test Conference (ITC), Fort Worth, TX, pp. 1-10, 2016, doi: 10.1109/TEST.2016.7805847.

[18] Z. Li, et al., "Fault Modeling, Structural Testing and Functional Testing," In: Micro-Electrode-Dot-Array Digital Microfluidic Biochips, Springer, 2019, doi: 10.1007/978-3-030-02964-7_4.

[19] F. Su, et al., "Testing of Droplet-based Microelectrofluidic Systems," Proceedings of the International Test Conference, pp. 1192-1200, 2003, doi: 10.1109/TEST.2003.1271108.

[20] F. Su, et al., "Ensuring The Operational Health of Droplet-based Microelectrofluidic Biosensor Systems," IEEE Sensors Journal, vol. 5, no. 4, pp. 763-773, 2005, doi: 10.1109/JSEN.2005.848127.

[21] F. Su, et al., "Concurrent Testing of Droplet-based Microfluidic Systems for Multiplexed Biomedical Assays," Proceedings of the International Test Conference, pp. 883-892, 2004, doi: 10.1109/TEST.2004.1387352.

[22] M. Zhu, et al., "A Built-In Self-Test Method for MEMS Piezoresistive Sensor," 2020 IEEE European Test Symposium (ETS), Tallinn, Estonia, pp. 1-6, 2020, doi: 10.1109/ETS48528.2020.9131587.

[23] Y. Miyake et al., "On-Chip Delay Measurement for Degradation Detection And Its Evaluation under Accelerated Life Test," 2020 IEEE 26th International Symposium on On-Line Testing and Robust System Design (IOLTS), Napoli, Italy, pp. 1-6, 2020, doi: 10.1109/IOLTS50870.2020.9159717.

[24] G. Hantos and M. Desmulliez, "Acoustic Methods for Detection of Specific Failure Modes in Capacitive MEMS Microphones," 2020 IEEE 8th Electronics System-Integration Technology Conference (ESTC), Tonsberg, Vestfold, Norway, pp. 1-8, 2020, doi: 10.1109/ESTC48849.2020.9229745.

[25] T. A. K. Gregory, "Enabling Technologies for Cultured Neural Network," Chapter 7, Academic Press, 1994.

[26] A. J. Bard and L. R Faulkner, "Electrochemical Methods," New York: John Wiley, 1980.

[27] D. A. Borkholder, "Cell Based Biosensors Using Microelectrodes," PhD Thesis, Electrical Engineering, Stanford University, 1998.

[28] R. S. C. Cobbold, "Transducers for Biomedical Measurements: Principles and Applications," Wiley, New York, 1974, doi: 10.1063/1.1134117. 


\section{BIOGRAPHIES OF AUTHORS}
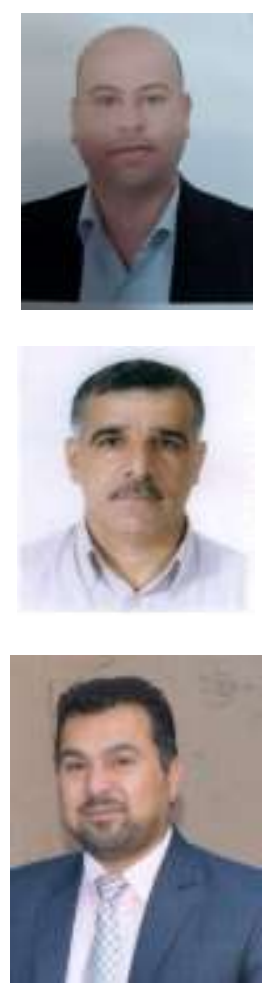

Qais Al-Gayem received the BS degree in Electrical and Electronic Engineering from the University of Babylon, and the MS degree in Electronic Engineering from the University of Technology, Iraq, in 1999 and 2001, respectively. Between 2002 and 2008, he worked as a lecturer in the Electrical Department, University of Babylon, Iraq. Following this, he studied for his $\mathrm{PhD}$ in the Engineering Department, Lancaster University, UK, and graduated in 2012. He is currently an Assistant Professor in the Electrical Department, Faculty of Engineering, University of Babylon, Iraq. His research interests include built-in-self-test (BIST) of MEMS and NEMS, health monitoring, and dependability in Bio-fluidic microsystems.

Hussain F. Jaafar received the BSc in electrical engineering from the University of Salahidden/ Iraq in 1981, the MSc in electronics engineering from the University of Technology/Iraq in 1984, and the PhD in Communication and Signal Processing from the University of Liverpool/ UK in 2012. He is currently Assistant Professor in Biomedical Engineering Department/ University of Babylon/ Iraq, His research interests include medical and biomedical engineering, medical informatics, machine learning, and pattern recognition

Asst. Prof. Dr. Saad S. Hreshee was born in Baghdad, Iraq, in 1974. He received his BS degree in Electrical Engineering from the Electrical Engineering Department, College of Engineering, University of Babylon, Iraq, in 1997. He obtained his MS degree in Electronic Engineering from the Electrical and Electronic Engineering Department, University of Technology, Iraq, in 2000, while his $\mathrm{PhD}$ in Electronic and Communication Engineering from the Electrical Engineering Department, College of Engineering, University of Basrah, Iraq, in 2007. Since 2001, he has been with the staff of the Department of Electrical Engineering, College of Engineering, University of Babylon, Iraq. For two years (2018-2020) he is the head of the electrical engineering department. His main research interests are antenna, signal processing, encryption and communication security. 